\title{
Pedunculopontine Nucleus--Rapid Eye Movement Sleep--Electroencephalogram--Desynchronization (PRED) Axis in the Evolution of Epilepsy: A Novel Concept
}

\section{Review}

Journal of Epilepsy Research pISSN 2233-6249 / elSSN 2233-6257

Received January 6, 2021

Revised February 28, 2021

Accepted March 7, 2021

Corresponding author:

Harinder Jaseja, MD

Vellore EEG Center, 8, C-Block,

Harishanker-puram, Lashkar, Gwalior

474002, India

Tel. +919425117588

E-mail; dr_jaseja@yahoo.com

\author{
Harinder Jaseja, MD \\ Vellore EEG Center, Gwalior, India
}

\begin{abstract}
Epilepsy is one of the commonest and oldest neurological diseases in the history of mankind, the exact pathophysiology of the evolution of which still remains elusive. The intimate and intriguing relation between epilepsy and sleep has been known for a long time. Rapid eye movement sleep (REMS) is well documented to exert potent antiepileptic action in human epilepsies and the underlying mechanism of which is largely based on its property to induce widespread electroencephalogram (EEG)-desynchronization. The pedunculopontine nucleus (PPN) owing to its property to enhance REMS has recently been under study for its potential role in intractable epilepsy (IE) and has been proposed as a novel deep brain stimulation target in IE. This brief paper unfolds the existing role of PPN, REMS, and EEG-desynchronization (PRED) in the evolution of epilepsy in an axial manner, the realization and comprehension of which is likely to open new avenues for further understanding of epileptogenesis, improved treatment of epilepsy and reducing the risk of IE. (2021;11:1-5)
\end{abstract}

Key words: Interictal epileptiform discharges, PRED axis, Intractable epilepsy

\section{Introduction}

Epilepsy is one of the commonest and oldest neurological diseases characterized by an enduring predisposition to unprovoked epileptic seizures and associated with neurobiological and social components. ${ }^{1}$ The prevalence of epilepsy in the general population is approximately $1 \%$, with an estimate of more than 60 million patients with epilepsy worldwide. The common hallmarks of epilepsy are interictal epileptiform discharges (IEDs), predominantly in the form of spikes and sharp waves that are displayed on an electroencephalogram (EEG), which is still considered a mainstay investigation for the detection of IEDs, therefore assisting in diagnosing an epilepsy state. ${ }^{2,3}$

Epileptogenesis has been described as a chronic, progressive and evolving process and is under intense investigation. ${ }^{4}$ With the advent of high-density EEG, magnetoencephalography (MEG) and functional magnetic resonance imaging (fMRI), epilepsy is now considered to be a network disorder involving interactions of large neuronal populations distant from one another. ${ }^{5}$ The heterogeneity of the epilepsy condition, the dynamics of molecular changes during epileptogenesis and the involvement of complex neural networks make it challenging to understand this phenomenon; therefore, the exact pathophysiol- ogy of epileptogenesis and evolution of epilepsy is still elusive. Recently, pedunculopontine nucleus (PPN) has been identified as a common denominator in regulating rapid eye movement sleep (REMS) on one hand andexerting antiepileptic influence on the other. ${ }^{6}$ In this brief paper, the role of PPN, REMS and EEG-desynchronization (PRED) in the evolution of epilepsy has been explored.

\section{REMS and Epilepsy}

The relation between epilepsy and sleep has remained intricate and intriguing and both are known to strongly influence each other. REMS exerts a strong antiepileptic influence on epileptogenetic process due to its property to induce widespread EEG-desynchronization, which constitutes its characteristic feature. ${ }^{7,8}$ Furthermore, increasing REMS with carbachol microinjection in the pontine nucleus has been shown to decrease the susceptibility to seizures in the electrogenic rat seizure model. ${ }^{9}$ It has even been proposed that REMS is an endogenous antiepileptic protection that humans are born with, akin to inherent immune system in the body; ${ }^{10}$ REMS has been suggested to be the most protective stage of sleep that possesses a strong antiepileptic effect against focal interictal discharges, focal seizures, and 
generalized seizures. ${ }^{11}$

There has been a growing trend in research on epilepsy and sleep architecture,especially in REMS, since long ago; the sleep architecture has been shown to be disrupted in patients with various forms of epilepsy. ${ }^{12-14}$ REMS shows phasic and tonic states and IEDs have been found to be strongly suppressed during phasic REMS. 15,16 Phasic REMS has an enhanced suppressive effect on IEDS, corroborating the role of EEG desynchronization in the suppression of interictal epileptic activity. ${ }^{15}$ The inhibitory effect on epileptic activity seems to be independent of the type of epileptic syndrome and location of epileptic activity and the pathological substrate. ${ }^{15}$ In a very recent prospective study, Hamdy et al., ${ }^{17}$ have concluded from their findings that REMS percentage and latency can control seizure attacks in idiopathic generalized epilepsy. A better understanding of neuronal processes involved during phasic REMS will help in understanding the mechanism of epileptogenesis and epilepsy and might assist in developing potential novel therapeutic strategies.

\section{EEG-Desynchronization and Epilepsy}

The mechanism of antiepileptic action of REMS is due to its ability to induce widespread EEG-desynchronization in the brain. ${ }^{7.18}$ Cortical desynchronization is an important and inherent characteristic feature of REMS, which strongly suppresses and may even abolish IEDs in the EEG. ${ }^{19}$ The importance of EEG-desynchronization can be understood from observations in patients with REMS behavior disorder (RBD), where EEG-desynchronization is impaired or dissociated and there is slowing of frequencies of EEG waves during REMS. ${ }^{20}$ Patients with RBD have been reported to exhibit a higher incidence of both epilepsy and IEDs; ${ }^{21,22}$ this increase in incidence of epilepsy and IEDs is apparently due to the loss of the protective influence of EEG-desynchronization. In both the above studies, ${ }^{21,22}$ there was a history of long standing RBD that had been either overlooked or misdiagnosed; the possibility of being overlooked or misdiagnosed has been found to occur commonly and has been highlighted in a study by Daly and Compton. ${ }^{23}$

In the study, Manni et al., ${ }^{21}$ argue that the long-standing RBD might have facilitated the occurrence of seizures as seizure facilitation has been documented in animal models of epilepsy with experimentally induced patterns of dissociated REM sleep with synchronized EEG ${ }_{r}^{24}$ whereas, in the second study, none of the study group patients had a neurodegenerative disease and the authors Manni and Terzaghi, ${ }^{22}$ hypothesize that epilepsy could be a result of reduction of seizure threshold due to REM sleep disruption. In yet another study, ${ }_{1}^{25}$ REM sleep without EEG desynchrony induced by pontine dissociationtechniques has been shown to result in the occurrence of epileptic phenomena in animal (cat) models of both generalized (penicillin) and focal epilepsy (kindling).

As mentioned earlier, impaired cortical activation/desynchronization has been hypothesized to occur during REM in RBD in an EEG spectral analysis study. ${ }^{20}$ The slowing of EEG background can raise the possibility of neurodegenerative changes in the study patients, but none of the study group patients had a neurodegenerative disease and the authors Manni and Terzaghi, ${ }^{22}$ hypothesize that epilepsy could be a result of reduction of seizure threshold due to REM sleep disruption and slowing of EEG background could be due to impaired cortical activation/desynchronization in $\mathrm{RBD}_{i}^{.20}$ thus, the authors Fantini et al., ${ }^{20}$ have suggested that chronically altered REM sleep, as seen in RBD, increases the likelihood of seizures in humans. It has also been proposed that EEG-desynchronization and strategic loss of connectivity during REMS reduces the likelihood of spatial and temporal summation of aberrant depolarizations, which accounts for its unique antiepileptic influence on seizures. ${ }^{11}$

REMS and EEG-desynchronization have also shown a noticeable association with intractable epilepsy (IE). A significant reduction in REMS has been reported in patients with drug-resistant epilepsy (IE) as compared to controls. ${ }^{26}$ Furthermore, the success and efficacy of adjunctive therapeutic procedures deployed in IE like temporal lobe resective surgery, vagal nerve stimulation and ketogenic diet have been found to be associated with increased REMS in responders with seizure control in comparison to non-responders. ${ }^{27-29}$ In view of its significant association with IE, EEG-desynchronization has also been advocated as a prospective biomarker of intractability in epilepsy. ${ }^{30}$

In earlier studies also, alertness and high frequency anterior thalamus stimulation have been reported to be associated with EEG-desynchronization and subsequent antiepileptic influence, ${ }^{31,32}$ on similar grounds, one study has demonstrated that $30-\mathrm{Hz}$ (high frequency) vagal nerve stimulation suppresses IEDs apparently via EEG-desynchronization while 5-Hz (low frequency) stimulation increases appearance of IEDs. ${ }^{33}$

\section{PPN and Epilepsy}

Deep brain stimulation (DBS) therapy is an acceptable and effective treatment strategy for patients with IE. Anterior thalamic nucleus (ATN) and centro-median nucleus (CMN) are the most commonly tar- 
geted sites for DBS in IE; ATN is functionally connected to limbic circuit and has been found to be more successful in controlling complex partial seizures as compared to generalized tonic clonic seizures, ${ }^{34}$ whereas, CMN-DBS appears to act via the reticular activating system and is more successful in intractable forms of generalized tonic-clonic seizures and Lennox-Gastaut syndrome, but has little or no influence on CPS. ${ }^{35,36}$

Recently, the PPN in brainstem has been under study and exploration for its potential role in epilepsy owing to its property of generating and enhancing REMS. ${ }^{37}$ Based on this property, it has even been proposed that PPN can be explored as a novel DBS target in IE. ${ }^{38,39}$ The antiepileptic action of PPN stimulation is supposedly via increasing REMS, which is suggested to be a natural inherent antiepileptic mechanism and can potentially prevent partial as well as generalized epilepsies, therefore, it could be superior to the conventional ATN and CMN sites for DBS in IE. ${ }^{6}$ The PPN is a part of REMS regulating networks; therefore, it is expected that the dysfunction of PPN may result in a decrease in REMS and subsequently increased susceptibility to seizures. Indeed, this has been reported in a recent voxel-based morphometry study ${ }^{40}$ in which atrophy of PPN was demonstrated in patients with sleep dominant seizures and PPN volume measured was significantly lower $(95.2 \pm 20.4 \mu \mathrm{L})$ in the patients with epilepsy in comparison to those in the healthy controls $(108.2 \pm 15.4 \mu \mathrm{L})$.

Several other recent studies have also demonstrated the antiepileptic potential of PPN. In a rat model of temporal lobe epilepsy, PPN has been shown to be strongly affected by kindling associated functional reorganization of neurocircuitry in the brain; ${ }^{41}$ whereas, in another study on rats, optogenetic stimulation of PPN during focal limbic seizures led to an increase in cortical gamma activity and a decrease in delta activity. ${ }^{42}$ Thus, although a few studies have suggested potential role of PPNS in animal models of epilepsy, no direct effect has yet been demonstrated in humans. However, in patients with temporal lobe epilepsy, functional connectivity disturbances have been reported in several structures including the PPN that recovered after successful epilepsy surgery. ${ }^{43}$

\section{The Novel Concept: The PRED Axis}

From the above discussion it is apparent that enhancement/ increase in REMS is associated with an increase in resistance to seizures; conversely, lack/decrease in REMS is associated with an increase in proneness to seizures. Moreover, dissociation of EEG-de- synchronization during REMS predisposes the patients to epilepsyas occurring in patients with RBD. ${ }^{21,22}$ Thus, from the above-cited studies and evidences, the PRED appear to play a significant role in the evolution of epilepsy in humans in an axial manner, which Jaseja conceives as PRED axis (comprising of PRED), something similar to the hypothalamo-pituitary-adrenal (HPA) axis hormonal regulatory system in humans. Disruption at any point in the PRED axis is therefore highly likely to increase proneness/susceptibility to epileptogenesis and cause epilepsy.

With the current pace of advances in technology for electrical and functional imaging of brain, studying the network abnormalities for epilepsy workup may not be very far in distant. It will not be irrational, therefore, to study and evaluate PRED axis in patients with epilepsy and consider therapeutic amendments if a disruption in the axis is suspected or observed to ensure optimal, effective and early control over seizure occurrence. This will serve as a novel approach towards effective management of epilepsy and even reduce the risk of development of intractability, keeping in view the large incidence of $\mathrm{IE}$, which amounts to as many as $30 \%$ of newly diagnosed patients with epilepsy.

The concept of PRED axis though presents a simplistic view of complex network interaction involved in epileptogenesis and epilepsy; nevertheless, it does lend an insight to the understanding of epileptogenesis in humans and can potentially stimulate further indepth and experimental studies to validate the significance of the axis in the evolution of epilepsy. The existence of PRED axis is basically and essentially hypothetical; it has been presented as a seminal postulation to stimulate further exploration and investigations and well designed studies are strongly recommended. The roles of PRED have been hypothesized to potentially operate in an axial manner akin to HPA axis in endocrinal system in humans, the realization of which can aid in early identification of an epilepsy state and may eventually assist in decreasing the incidence of IE.

\section{Conflict of Interest}

The author declares that (s)he have no conflicts of interest.

\section{References}

1. Fisher RS, Acevedo C, Arzimanoglou A, et al. ILAE official report: a practical clinical definition of epilepsy. Epilepsia 2014;55:475-82.

2. Rosenow F, Klein KM, Hamer HM. Non-invasive EEG evaluation in epilepsy diagnosis. Expert Rev Neurother 2015;15:425-44. 
3. Feyissa AM, Tatum WO. Adult EEG. Handb Clin Neuro/ 2019;160:103-24.

4. Pitkänen A, Lukasiuk K, Dudek FE, Staley KJ. Epileptogenesis. Cold Spring Harb Perspect Med 2015;5:a022822.

5. Laufs $H$. Functional imaging of seizures and epilepsy: evolution from zones to networks. Curr Opin Neurol 2012;25:194-200.

6. Jaseja H. Deep-brain stimulation in intractable epilepsy: pedunculopontine nucleus versus thalamic nuclei: a perspective. World Neurosurg 2014;82:e568-9.

7. Shouse MN, Farber PR, Staba RJ. Physiological basis: how NREM sleep components can promote and REM sleep components can suppress seizure discharge propagation. Clin Neurophysio/ 2000;111 Suppl 2:S9-18.

8. Shouse MN, Siegel JM, Wu MF, Szymusiak R, Morrison AR. Mechanisms of seizure suppression during rapid-eye-movement (REM) sleep in cats. Brain Res 1989;505:271-82.

9. Kumar P, Raju TR. Seizure susceptibility decreases with enhancement of rapid eye movement sleep. Brain Res 2001;922:299-304.

10. Jaseja H. Purpose of REM sleep: endogenous anti-epileptogenesis in man -- a hypothesis. Med Hypotheses 2004;62:546-8.

11. Ng M, Pavlova M. Why are seizures rare in rapid eye movement sleep? Review of the frequency of seizures in different sleep stages. Epilepsy Res Treat 2013;2013:932790.

12. Gigli GL, Calia E, Marciani MG, et al. Sleep microstructure and EEG epileptiform activity in patients with juvenile myoclonic epilepsy. Epilepsia 1992;33:799-804.

13. Maganti $R$, Sheth RD, Hermann BP, Weber $S$, Gidal BE, Fine J. Sleep architecture in children with idiopathic generalized epilepsy. Epilepsia 2005;46:104-9.

14. Halász P, Terzano MG, Parrino L. Spike-wave discharge and the microstructure of sleep-wake continuum in idiopathic generalised epilepsy. Neurophysiol Clin 2002;32:38-53.

15. Campana C, Zubler F, Gibbs $S$, et al. Suppression of interictal spikes during phasic rapid eye movement sleep: a quantitative stereo-electroencephalography study. J Sleep Res 2017;26:606-13.

16. Frauscher B, von Ellenrieder N, Dubeau F, Gotman J. EEG desynchronization during phasic REM sleep suppresses interictal epileptic activity in humans. Epilepsia 2016;57:879-88.

17. Hamdy MM, Elfatatry AM, Mekky JF, Hamdy E. Rapid eye movement (REM) sleep and seizure control in idiopathic generalized epilepsy. Epilepsy Behav 2020;107:107064.

18. Jaseja H. Mechanism of endogenous anti-epileptogenesis during rapid eye movement sleep. Med Hypotheses 2006;66:866.

19. Herman ST, Walczak TS, Bazil CW. Distribution of partial seizures during the sleep--wake cycle: differences by seizure onset site. Neurology 2001;56:1453-59.

20. Fantini ML, Gagnon JF, Petit D, et al. Slowing of electroencephalogram in rapid eye movement sleep behavior disorder. Ann Neurol 2003;53: 774-80.

21. Manni R, Terzaghi M, Zambrelli E. REM sleep behavior disorder and epileptic phenomena: clinical aspects of the comorbidity. Epilepsia
2006;47 Suppl 5:78-81.

22. Manni R, Terzaghi M. REM behavior disorder associated with epileptic seizures. Neurology 2005;64:883-4.

23. Daly JJ, Compton SA. Rapid eye movement (REM) sleep behaviour disorder; an easily missed diagnosis, a readily treatable condition. Ulster Med J 2002;71:62-5.

24. Shouse MN. Mechanisms of sleep and arousal: relationship to epilepsy. In Bazil CW, Malow BA, Sammaritano MR, ed. Sleep and epilepsy: the clinical spectrum. Amsterdam: Elsevier Science B.V., 2002;93-107.

25. Shouse MN, King A, Langer J, et al. Basic mechanisms underlying seizure-prone and seizure-resistant sleep and awakening states in feline kindled and penicillin epilepsy. In: Wada JA, ed. Kindling 4. New York: Plenum Press, 1990;313-27.

26. Pereira AM, Bruni O, Ferri R, Palmini A, Nunes ML. The impact of epilepsy on sleep architecture during childhood. Epilepsia 2012;53:1519-25.

27. Hallböök T, Köhler S, Rosén I, Lundgren J. Effects of ketogenic diet on epileptiform activity in children with therapy resistant epilepsy. Epilepsy Res 2007;77:134-40.

28. Fernández-Guardiola A, Martínez A, Valdés-Cruz A, Magdaleno-Madrigal VM, Martínez D, Fernández-Mas R. Vagus nerve prolonged stimulation in cats: effects on epileptogenesis (amygdala electrical kindling): behavioral and electrographic changes. Epilepsia 1999;40:822-9.

29. Serafini A, Kuate $C$, Gelisse $P$, et al. Sleep before and after temporal lobe epilepsy surgery. Seizure 2012;21:260-5.

30. Jaseja $H$. Intractability in epilepsy: role of EEG desynchronization in early identification. Clin EEG Neurosci 2015;46:266-7.

31. Fenwick PB. The relationship between mind, brain, and seizures. Epilepsia 1992;33 Suppl 6:\$1-6.

32. Hodaie M, Wennberg RA, Dostrovsky JO, Lozano AM. Chronic anterior thalamus stimulation for intractable epilepsy. Epilepsia 2002;43:603-8.

33. Olejniczak PW, Fisch BJ, Carey M, Butterbaugh G, Happel L, Tardo C. The effect of vagus nerve stimulation on epileptiform activity recorded from hippocampal depth electrodes. Epilepsia 2001;42:423-9.

34. Fisher $R$, Salanova V, Witt $T$, et al. Electrical stimulation of the anterior nucleus of thalamus for treatment of refractory epilepsy. Epilepsia 2010; 51:899-908.

35. Velasco F, Velasco AL, Velasco M, Jiménez F, Carrillo-Ruiz JD, Castro $G$. Deep brain stimulation for treatment of the epilepsies: the centromedian thalamic target. Acta Neurochir Supp/ 2007;97(Pt 2):337-42.

36. Valentín A, García Navarrete E, Chelvarajah $R$, et al. Deep brain stimulation of the centromedian thalamic nucleus for the treatment of generalized and frontal epilepsies. Epilepsia 2013;54:1823-33.

37. Lim AS, Moro E, Lozano AM, et al. Selective enhancement of rapid eye movement sleep by deep brain stimulation of the human pons. Ann Neurol 2009;66:110-4.

38. Jaseja $H$. Pedunculopontine nucleus stimulation: a novel adjunctive therapeutic approach in intractable epilepsy. Epilepsy Behav 2013;27:279.

39. Liu TT, Feng J, Bu HL, Liu C, Guan XH, Xiang HB. Stimulation for the compact parts of pedunculopontine nucleus: an available therapeutic 
approach in intractable epilepsy. Epilepsy Behav 2013;29:252-3.

40. Cho KH, Cho YJ, Lee Bl, Heo K. Atrophy of the pedunculopontine nucleus region in patients with sleep-predominant seizures: a voxel-based morphometry study. Epilepsia 2016;57:e151-4.

41. Nolte MW, Löscher W, Gernert M. Pedunculopontine neurons are involved in network changes in the kindling model of temporal lobe epilepsy. Neurobiol Dis 2006;23:206-18.
42. Furman M, Zhan Q, McCafferty C, et al. Optogenetic stimulation of cholinergic brainstem neurons during focal limbic seizures: effects on cortical physiology. Epilepsia 2015;56:e198-202.

43. González HFJ, Goodale SE, Jacobs ML, et al. Brainstem functional connectivity disturbances in epilepsy may recover after successful surgery. Neurosurgery 2020;86:417-28. 